\title{
Student Perceptions on Learning - Inside and Outside Classrooms
}

\section{Miss Sreyoshi Bhaduri, Virginia Polytechnic and State University}

Sreyoshi Bhaduri is a Ph.D. candidate at Virginia Tech Department of Engineering Education. She is a proponent for use of technology in the classroom as well as education research. Sreyoshi is a Mechanical Engineer by training, who likes programming and algorithms to make life easier and more efficient. For her doctoral dissertation, she is exploring ways in which machine learning algorithms can be used by instructors in engineering classrooms.

\section{Dr. Holly M. Matusovich, Virginia Polytechnic Institute and State University}

Dr. Matusovich is an Assistant Professor and Assistant Department Head for Graduate Programs in Virginia Tech's Department of Engineering Education. She has her doctorate in Engineering Education and her strengths include qualitative and mixed methods research study design and implementation. She is/was PI/Co-PI on 8 funded research projects including a CAREER grant. She has won several Virginia Tech awards including a Dean's Award for Outstanding New Faculty. Her research expertise includes using motivation and related frameworks to study student engagement in learning, recruitment and retention in engineering programs and careers, faculty teaching practices and intersections of motivation and learning strategies. Matusovich has authored a book chapter, 10 journal manuscripts and more than 50 conference papers. 


\title{
Student Perceptions on Learning -Inside and Outside Classrooms
}

\begin{abstract}
Research show that engineering students need to develop more accurate conceptual understanding. Doing so requires the motivation to engage in the difficult work of developing conceptual understanding which often requires more effective learning strategies than those that students currently use. Although prior studies have attributed learning to motivation based on theories for interest, relatedness, usefulness, or changes over time, we wanted to approach our data from a more open and emergent standpoint, and have our findings grounded in the views of our participants. In this research study we wanted to understand student learning in settings such as those related to hobbies, sports, or other interests for which students describe having engaged in the activity of learning and compare our findings to learning in academic settings. We analyzed transcripts from semi-structured interviews with 17 engineering students. The interview was part of a larger study to understand engineering specific learning strategies. We see similarities in the behavioristic approach to learning through practice and reward in both the settings. However, important to note are the differences in learning in the two settings. We have described how engineering students often seem to value the coach or expert as a role model in non-academic settings, however not many students seemed to equate faculty or academic coaches to serve as possible role models. Multiple students mentioned technology in helping them understand techniques and principles outside the classroom. However, the role of technology to aid in learning for concepts relevant inside the classroom was only highlighted by two participants. We believe that the findings from this research can inform engineering educators on successful strategies employed by engineering students in non-academic settings, and this in turn may be useful to incorporate in academic settings to improve student engagement and hence conceptual understanding.
\end{abstract}

\section{Introduction}

Existing engineering education research has focused both on the development of methods to assess conceptual knowledge in specific topics such as statics (e.g. Steif and Dantzler, 2005) or thermodynamics (e.g. Streveler, Miller, Santiago-Román, Nelson, Geist and Olds, B. M, 2011), as well as on how learners understand concepts (e.g. Vosniadou, 2007). However, few researchers have looked into the intentional ways in which engineering students engage in learning. Helping students develop effective learning strategies have been found to be useful for them in accomplishing their conceptual learning goals (Bransford, et al., 2002). Thus, research focusing on learning strategies adopted by students may be useful in helping students with conceptual learning.

To better understand the learning among engineering students in academic settings, we decided to look at how students learn in non-academic settings. In this case, non-academic settings refer to hobbies, sports or interests that students have, and for which they describe having engaged in the activity of learning. Specifically, we wanted to examine settings in which students enjoy learning. We believe that an understanding of the strategies employed by students in non-academic settings, may be useful to help them learn in academic settings. Although prior studies have attributed learning to motivation based on theories for interest, relatedness, usefulness, or changes over time (e.g. Eccles, \& Wigfield, 2002), we wanted to approach our data from a more open and emergent standpoint. That is, we did not want to pick one framework 
and test applicability but rather focus on emergent findings grounded in the views of our participants. Thus, our study uses a Grounded Theory method (Charmaz, 2006) to understand learning in non-academic settings and compare that to learning in academic settings, based on the views expressed by the students.

The purpose of our study is thus to use Grounded Theory method to gain a deeper understanding of how the process of learning in non-academic settings compares to learning in academic settings, for undergraduate engineering students. The research questions driving this qualitative study are:

1. Based on the students' responses, what strategies helped them learn in settings outside the classroom?

2. What causal mechanism emerged from the data as being responsible for helping students learn in settings outside the classroom?

3. From the students' perspective, how does learning in non-academic settings differ from learning in academic settings?

To the answer these questions, we analyzed transcripts from semi-structured interviews with 17 engineering students.

\section{Sensitization}

With regards to use of theory, classical Grounded Theory researchers advocate delaying comprehensive use of literature until after the entire story has emerged from empirical data (Glaser, 1978; Glaser \& Strauss, 1967). However, a more pragmatic approach to use of literature and theory is offered by Lempert (2007) in terms of sensitizing concepts, stating that literature reviews alert a researcher of the gaps in existing literature, which can aid in theorizing and lead to a more nuanced story.

To help situate this research study, we draw primarily from two bodies of literature. The first area is literature on the importance of conceptual understanding, and learning strategies specific to students in engineering classrooms. For this we also draw from motivational theories related to student learning. We believe that this discussion will help situate the need for this research by identifying the unique learning strategies employed by engineering students, understanding existing theories explaining the choice for these strategies, and finally, recognizing the importance of modifying instructional intervention to further engage students by building upon those learning strategies. We believe that results of the analysis of this research will help inform engineering educators on successful strategies employed by engineering students in non-academic settings, and this in turn may be useful to incorporate in academic settings to improve student engagement and hence conceptual understanding. The second area of literature that we will present is a background on the Grounded Theory method which guided our analysis.

\section{Learning Strategies, Motivation and Conceptual Understanding}

Researchers have identified that a limited understanding of concepts by undergraduate students graduating into the workforce is a challenge facing STEM educators (Rittle-Johnson, Siegler and Alibali, 2001). Specific to engineering, research shows that graduating students struggle with misconceptions about real world applications and concepts in statics, electricity and magnetism, and thermodynamics, as they move into industry (Strevler, Litzinger, Miller and 
Steif, 2008). Conceptual knowledge in engineering topics including definitions and applications is crucial to practicing engineers, since deeper understanding of concepts will ultimately help students progress to become experts in their respective domains (Bransford et al., 2000). Lack of such conceptual knowledge may lead to students formulating deep misconceptions that can be difficult to correct (e.g. Streveler et al., 2008). However, research can help in identifying the reason behind the formation of misconceptions, to help students develop learning strategies to strengthen conceptual understanding in the classrooms. Much of such research has occurred from a cognitive perspective, i.e., learning occurs through the construction of mental models (Newstetter \& Svinicki, 2014; Phillips \& Soltis, 2004). However, theories on learning also suggest a need to consider what prompts learners to engage in learning (e.g., Linnenbrink \& Pintrich, 2002; Pintrich, Marx, \& Boyle, 1993).

Existing theories of motivation can offer one way to understand learner intentionality, or why students adopt specific learning strategies. Johri and Olds (2011) state that in a cognitive framework, student motivation is intrinsic, meaning that the motivation to perform a task comes from within an individual, rather than extrinsic rewards such as grades. Theories of motivation focus primarily on the relation of beliefs, values and goals with action (Eccles and Wigfield, 2002). For example, competence beliefs refer to an individual's expectancy of how one will perform on a given task (eg. "Can I do this?" (Matusovich et al., 2010). Values relate to an individual's reasons or incentives for performing a task ("Do I want to do this?" (Matusovich et al., 2010)), and goals (What will I accomplish at the end of doing this?) may refer to the objective sought at the end of completion of task (Eccles and Wigfield, 2002). Multiple constructs may be integrated to better understand the engineering students' learning experience. For instance, Jones et al. (2010) suggests that both expectancy and value related constructs are useful in studying the motivations of undergraduate engineering students, and that integrating constructs and studying the interactions among factors may help examine more closely the student experiences in engineering classrooms. In fact, Jones created the MUSIC model of motivation which integrates a variety of motivation theories (Jones, 2009).

A sensitization on topics related to motivation, learner intentionality, and cognition was important for this research to advance a more nuanced explanation of the emergent themes which were grounded in the participants' views. Although we kept these concepts in mind, we focused on emergent findings through Grounded Theory.

\section{Grounded Theory Method}

Grounded Theory is most popularly used to learn about the causal relationships involved in a process (Charmaz, 2006). A causal relationship explains the whys and hows for a process, while a process itself consists of unfolding temporal sequences that may have identifiable markers with clear beginnings and endings (Charmaz, 2006). The selection of any research design should be informed by the research question that is being asked (Borrego et al., 2009). In this case the purpose of this research was to develop a deeper understanding of the process of learning adopted by students inside and outside the classroom. Thus, Grounded Theory seemed most appropriate as a choice of method to form a deeper understanding grounded in the views of the participants particularly given the many different and, at times conflicting, models of learning and motivation. The researchers who adopt Grounded Theory have different theoretical perspectives depending on their views on objectivity of the researcher, their world views, and on the use of theory. For instance classical "Glasserian" Grounded Theory focussed on objectivity and prescribed researcher neutrality emphasizing that the theory will form from the data and will 
be grounded in the views of the participants (Glaser, 1978). However, absolute empathetic neutrality may be a utopic construct for researchers working on specific problems and addressing similar challenges in education. Lempert (2007) offers a more pragmatic explanation of Grounded Theory research and describes it as a negotiation between the researcher and the research participants. Thus, she describes Grounded Theory research as a "practice of give and take" which is an unplanned process of continuous negotiation by all participants in the research process including the researcher (pp.248). Through the course of this study we drew the most from a more pragmatic approach to conducting Grounded Theory research. We will explain in detail our researcher positionality in the Methods section of this paper.

\section{Method}

This study is a qualitative secondary analysis (Heaton, 2004) of existing interview data, using Grounded Theory method. The data used in the larger, primary analysis were collected as part of a NSF-funded study related to conceptual understanding among engineering students. Charmaz (2006) states that the interview process is a good fit for Grounded Theory since like Grounded Theory, intensive interviews have the characteristics of being open-ended yet directed, shaped yet emergent, paced yet unrestricted". Charmaz (2006, pp.28) also recommends the use of an additional method to complement the interviewing process such as requesting participants to document their thoughts. However, since we worked with pre-collected data, we were unable to request participants to document deeper or more insightful thoughts, or influence the original data collection process in any way. The primary study of which this analysis is part of, has been approved by the Institution Review Board (IRB) and this analysis is in accordance with the recommended protocol for analysis involving human subjects. To help maintain participant anonymity to protect their identities, we continued the secondary analysis with pseudonyms for each school, course and participant.

\section{Site}

The sites at which the interviews were conducted were initially chosen to enable diverse contexts to ease comparison and contrasts across participant views (Morelock, Matusovich, Cunningham, \& Hermundstad, 2016). The first research site (PubU) was a large, public, research-focused university in the Mid-Atlantic United States (PubU). The second research site (PriU) was a small, private not-for-profit, teaching-focused university in the Midwestern United States. The student population at PriU was less than 5000 (approximated at the time of the interview), while that for PubU was larger at approximately 35,000. A comparative description of the sites is available from Morelock et al. (2016) to provide an overview of the differences between the two sites.

Table 1: Comparison of characteristics of both the sites *Data for these characteristics were derived from The Carnegie Classification of Institutions of Higher Education in August 2015. Student population was approximated to protect institutional anonymity.

\begin{tabular}{|c|c|c|}
\hline Characteristic & PriU & PubU \\
\hline Control & Private not-for-profit* & Public* \\
\hline Institutional Focus & Teaching & Research* \\
\hline Student Population & $<5,000^{*}$ & $<35,000 *$ \\
\hline Undergraduate Curricular Focus & Engineering* & Arts \& Sciences* \\
\hline U.S. Geographical Region & Midwest & Mid-Atlantic \\
\hline $\begin{array}{l}\text { Engineering students select an engineering major at } \\
\text { the start of their... }\end{array}$ & $1^{\text {st }}$ Year & $2^{\text {nd }}$ Year \\
\hline
\end{tabular}




\section{Participants}

We analyzed responses to semi-structured interviews with mechanical engineering students at two universities. Although a total of 21 students were interviewed, we present the analysis for 17 students as these are the student who completed the entire interview protocol within the designated time allotted. The students from both the universities were part of a particular course and were invited via email to participate in the study. Since the courses were for different years at both the universities, the participants were from different years as well. We analyzed responses for ten students in their second year at PriU and seven students in their third year at PubU. The Table 2 below provides an overview of the participants.

Table 2 Participant Overview with Pseudonym and Gender

$\begin{array}{cccc}\text { Pseudonym } & \text { Gender } & \text { Pseudonym } & \text { Gender } \\ \text { Jerry } & \text { M } & \text { Luis } & \text { M } \\ \text { Lisa } & \text { F } & \text { Hunter } & \text { M } \\ \text { Walter } & \text { M } & \text { Greg } & \text { M } \\ \text { Jenna } & \text { F } & \text { Robert } & \text { M } \\ \text { Joseph } & \text { M } & \text { Justin } & \text { M } \\ \text { Sarah } & \text { F } & \text { Chris } & \text { M } \\ \text { Becca } & \text { F } & \text { Chelsea } & \text { F } \\ \text { Jeffrey } & \text { M } & & \\ \text { Brad } & \text { M } & & \\ \text { Tom } & \text { M } & & \\ & & & \end{array}$

\section{Data Collection and Analysis}

We used a semi-structured interview protocol (Patton and Patton, 2002), which had been developed to gain insight on engineering student learning strategies and conceptual understanding. In this section, we will present the results of our analysis of the responses to the questions on the interview protocol which focused on asking the participants about an activity they enjoyed and how they learned in the settings outside the classroom for that activity. Students were then asked to reflect on how the learning in both the settings (ie. inside the classroom and outside) were different or similar.

For interviews at PriU we asked:

"Tell me something you enjoy doing. How did you go about learning how to do that? (examples might be an instrument, sport, language, game, cooking, or other craft) How is learning for this activity the same as and different than learning in academic classes? What makes it different?"

For interviews at PubU, we asked:

"Tell me something you enjoy doing. How did you go about learning how to do that? (examples might be an instrument, sport, language, game, cooking, or other craft); How is learning for this activity the same as learning in academic classrooms?; How is learning for this activity different than learning in academic classes?; What makes it different?"

Based on our research questions, we extracted the sections which were responses to the questions of interest from the transcripts and conducted an open and emergent analysis following 
the steps prescribed in exemplar Grounded Theory Literature (eg. Charmaz, 2006; Glaser, 1978; Glaser \& Strauss, 1967). Thus, we first started with open coding line by line for the data set. This open coding lead to creation of fractures and labeling data. Once we had completed our open coding we continued onto axial coding of making connections between categories and subcategories, which had emerged from our previous round of open coding. Finally, we completed selective coding which is an integrative process of selecting core categories and organizing the relationship among all emerged categories.

The categories developed from the code were used to develop a paradigm model to help understand the process of learning for non-academic settings. Throughout the process we practiced regular memo-ing and bracketing our biases. Theory and existing literature influenced our final memo. Finally, we found our negative cases to confirm the proposed causal mechanism to support our model on learning outside the classroom. The Figure 1 below shows the iterative nature of grounded theory research, and elaborates on the steps we followed to determine the causal mechanism to explain our paradigm model. In the figure, the double sided arrows suggest the iterative nature of the steps in conducting Grounded Theory research.

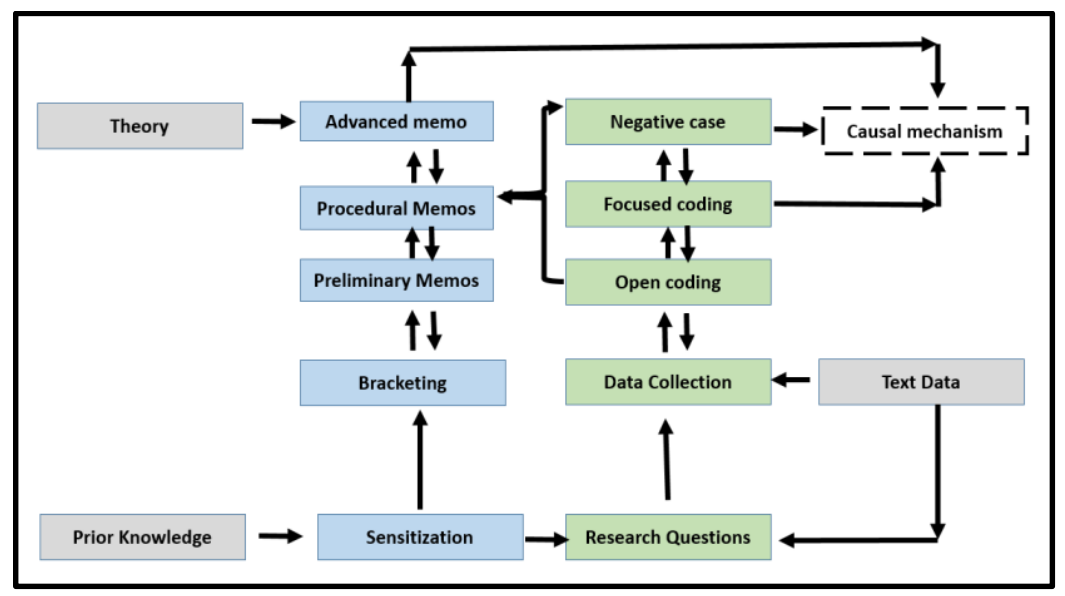

Figure 1:

Iterative steps in data collection and data analysis followed in this study based on recommended steps described in exemplar Grounded Theory literature (eg. Charmaz, 2006; Glaser, 1978; Glaser \& Strauss, 1967).

Figure inspired by the visual representation of Grounded Theory presented by Charmaz (2006, pp.18)

\section{Researcher Positionality}

In conducting Grounded Theory research, researchers may subscribe to various descriptions of researcher positionality based on the school of thought they identify the most with (Urquhart and Fernandez,2013). Both researchers of this analysis identify with the pragmatic worldview, which as described by Creswell (2014) is one in which the researcher does not subscribe to any particular method or world-view. Rather, the researcher uses a "what works" approach to conduct the research in an attempt to best analyze the problem (p.11). Thus, most of the choices made in conducting this research will be informed by a pragmatist stance to conducting Grounded Theory, and follows rationale provided by Lempert (2007), although they may be influenced in parts by works of both Glasser (1978) and Charmaz (2014)

\section{Quality of research}

We engaged in several practices to promote the quality of this research. Leydens et al. (2004) recommend clarifying researcher bias through regular memoing, and providing dense description in a study to help establish quality of the research. Lempert(2007) cites Charmaz (2006) and suggests that the activity of memoing "roots researcher in the analyses of data while 
simultaneously increasing the level of abstraction of his/her analytical ideas" (pp.245). For this research we conducted regular and detailed memo-ing of the choices and through the analyses. The results section of this report is an extension of the detailed memoing conducted through the course of the research. In addition to regular memoing during the research process, and providing dense description in this report to clarify our biases to the reader, we also used peer-debriefing to discuss results of analysis and interpretation. Thus, the researchers met with peers in engineering education at regular intervals through the period of analysis, to describe the process and the rationale behind certain codes and themes which generated from the data.

\section{Limitations}

Since this was a secondary analysis of interview transcripts, one of the major limitations of this research arises from the nature of the pre-collected data. As described earlier, the interview protocols used at the two sites were slightly different. However, the section that was analyzed for this study were responses to questions which were similar in both the sites, although at different stages of the interview. In general, we found the responses in terms of describing the activity, and perceived similarities and differences among both the settings seemed to be comparable across both the sites. However, it did seem that since the students at PubU answered this question towards the end of the interview, they anticipated having to draw on similarities and differences in learning across both settings. For instance, a participant from PubU remarks: "I knew that was going back somehow", when asked about comparing learning in non-academic settings to learning in the classroom. The hour long interview on learning and conceptual understanding in engineering thus may have contributed to students at PubU reflecting more on the act of learning in both the settings and hence providing richer and more descriptive responses to the questions asking them about comparisons across the settings.

Another limitation is the number of participants whose views were analyzed. A minimum of 25 interviews followed by a second theoretical sample is suggested in literature, to help Grounded Theory research to be truly grounded in participant views (Creswell, 2014; Tashakkori $\&$ Teddlie, 1998). However, we had access to responses of only 17 participants. Although we did reach a certain level of saturation in terms of codes and themes which generated from our existing dataset, further round of theoretical sampling may be needed to completely establish a more complete paradigm model.

\section{Results and Discussion}

Our findings suggest that although some similarities do exist, engineering students mostly view learning in academic and non-academic settings very differently. In this section we use our results of analysis to answer the broader research questions which drove the analysis. As specified earlier, our research questions are: 1. Based on the students' responses, what specific strategies helped them learn in settings outside the classroom? 2. What causal mechanism emerged from the data as being responsible for helping students learn in outside the classroom settings? 3. From the students' perspective, how does learning in non-academic settings compare to learning in academic settings?

\section{RQ1: Learning Strategies Adopted in out-of-class settings}

The analysis of the responses revealed four primary strategies which students adopted to help them learn in settings outside the classroom: Practicing, Using Technology, Observing Experts 
or Role Models, and Working with Others. In addition to these four, multiple participants also explained how a combination of primary strategies helped them learn.

Practicing. Practicing emerged as the most dominant strategy, and fifteen students out of the total seventeen highlighted the importance of practice in learning in settings outside the classroom. For example, Luis from PriU emphasized practice being his most important strategy in learning to play basketball. Greg, also from PriU reflected on his strategy while learning to play the guitar and commented on the success due to relentless practice. He commented: "It was such slow progress at first, it was really frustrating. I don't know, I'll just work at something, just practice pretty relentlessly until it starts to make sense."

Using Technology. Students valued the role of technology in helping them learn in nonacademic settings. Out of the 17 participants, five described using technology in learning for their hobby. For example, Hunter from PriU reflects on the role of technology in him building a computer from scratch:

"Everything I learnt, and did, finding the right parts for the right prices, making sure I took all the necessary precautions, I learnt from sites like Reddit or random forums off of Google."

Similarly, Jerry from PubU noted the benefits of blogs and online forums to help him in his hobby of water fowl hunting. Chris attributes learning from online resources similarly and explained how he applies them to understand the workings of different hobbies such as photography. He noted:

"Once I got past the whole, I suppose, I guess you could call it the honeymoon phase of the hobby, I started looking at the more technical aspects of it, so I just started looking up, "all right, why ..." Again, the reasons why. I feel that if we could find the reasons why, you will know how. I always just enjoyed going to the internet, join forums, ask questions on message boards and such, and then you just pick it up and try it out."

Observing Experts. Strategies related to being inspired by experts or learning through observing experts emerged through analysis of the responses. Ten participants described instances where they were inspired to take up a hobby by experts or learnt through observing them. For example, Lisa from PubU reflected on taking up Zumba dancing as a hobby, and explained how she followed the steps by the teacher or the expert in the classroom to help with learning of the steps. Similarly, Luis from PriU reflected on how watching professionals play basketball helped him learn and remarked "I watch a lot of people play basketball and try to imitate what they do, imitate what works."

Working with Others. Four participants described how they pursued the hobby while working with others. Lisa from PubU also recalled following along with the steps of other dancers in her Zumba group. Similarly, Greg from PriU explained how playing soccer alone can usually get monotonous, and how he usually played with friends and learnt from them.

Pairing multiple strategies. Pairing multiple strategies also emerged as a popular learning strategy based on the student responses. Out of the seventeen participants, nine explained how a combination of strategies helped them learn their hobbies. Explaining about the importance of being inspired by a role model as well as observing experts, paired with practice. 
Pairing with practice seemed most dominant of the multiple strategies. Luis from PriU explains how he learnt to play basketball:

"Definitely watching the people or watching on TV, whatever it is, gives you the idea of what to do, but you're not going to know how to do it until you actually go practice it. I feel like the watching gives you the idea. My dad gave me the inspiration for it. The practicing gives me the actual application of those first two things, the inspiration and the idea of what I need to work on."

Similarly, Hunter also from PriU, mentioned using technology to seek answers or tutorials on a wide range of hobbies and interests including exercising, working on his car, and building a computer. However, he acknowledged turning to friends for answers not available easily online, thus demonstrating use of multiple strategies to help him achieve his goal of learning.

\section{RQ2: Emergent Causal Mechanism Supporting Learning Outside the Classroom}

To answer the second research question, we present our paradigm model which emerged from the data. Paradigm models are theoretical models, which emerge as a result of Grounded Theory analysis. The main components of the model include constructs like causal condition or causal mechanism which help us understand the phenomenon that we are interested in better. In this case our phenomenon is: learning outside the classroom and the context is related to undergraduate engineering students engaging in activities outside the classroom. Based on exemplar Grounded Theory research the paradigm model draws relationships between the causal conditions (ie. the conditions that influence the phenomenon), the causal mechanism suggesting when casual conditions influence a phenomenon, the context and the intervening conditions all of which usually emerge from the participant views and affect the strategies that are used to bring about certain consequences or outcome (Charmaz, 2006). In this case the outcome could be thought of as expertise in an activity (outside the classroom).

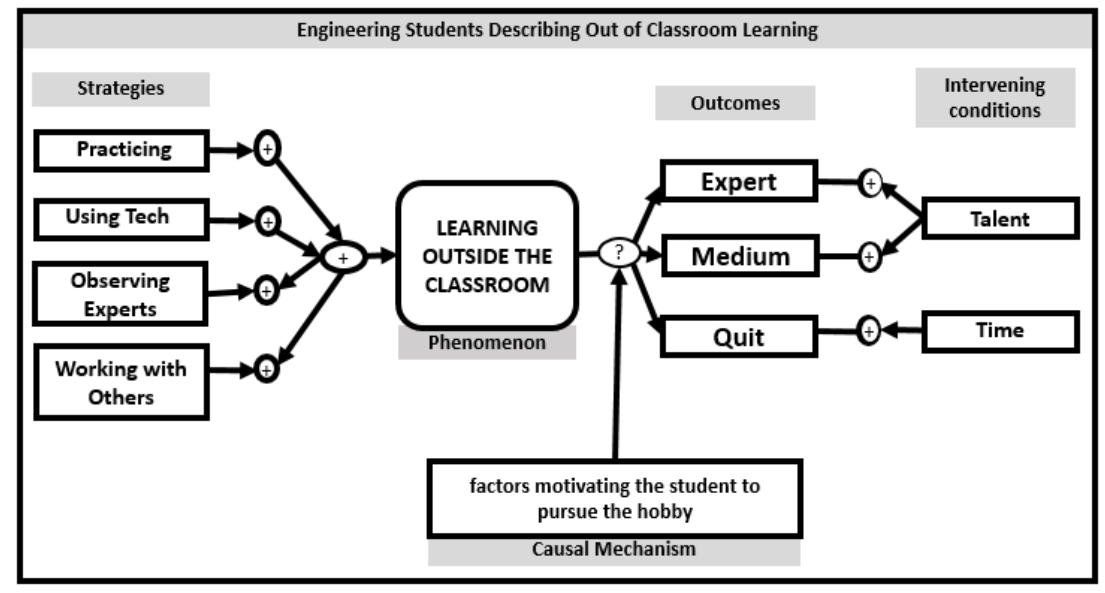

Figure 2:

Grounded Theory Model

based on student descriptions of learning

From Figure 2 above we can see that the phenomenon of interest at the center is the learning that happens in out-of-class settings. Based on our analysis of the student responses, this phenomenon of learning is influenced primarily by four major learning strategies or a combination of the strategies.

The process of learning ultimately results in participants either gaining engagement in the hobby or activity. The engagement is depicted in three levels: being actively engaged, being average performers, or entirely quitting the hobby. The levels of engagement with the hobby may 
be influenced by the intervening conditions. For example, a participant elaborated on how due to lack of time they had to stop pursuing their hobbies altogether. Another participant reflected on how they were not talented enough to ever gain expertise in soccer, which was their hobby of choice. Talent or perceptions of talent may also be considered as an intervening condition which leads to mastery of an activity or hobby. For example, a participant elaborated on how practice and talent had made them an expert at drawing, which they had taken up as a hobby.

The causal mechanism explaining the phenomenon of learning and the related outcomes can be thought of in terms of the factors motivating the student to pursue the hobby. Consider as the negative case the experiences of Greg from PriU who reflected on how the slow progress at the beginning was frustrating, but little success along the way lead to motivation and increased confidence. On the importance of learning through experiencing small successes in playing the guitar, Greg commented:

"You start really slowly and it doesn't sound good at all, but eventually you get the calluses on your fingers, you work over time, you get more coordination, you learn how to read tablature on the internet, that's what I learned from. Then you just get more confidence in yourself and it just builds on itself."

This negative case serves to re-affirm that the causal mechanism is closely related to motivational constructs such as self-efficacy. Had the small successes not lead to building confidence and interest, Greg would have likely quit learning to play the guitar.

\section{RQ3: Comparisons Across the Settings}

The analysis of the responses revealed four important factors on which students compared learning across both the settings. The dominant bases for comparison were Need for Practice and Motivation Related Comparisons. Fewer participants elaborated on Expertise Related Comparisons, and Use of Technology in both settings.

Need for Practice. Practice seemed to be the most dominant similarity which emerged based on the student responses to comparing learning strategies across both the settings. Nine out of seventeen students related practice as an important strategy in both in and out of class settings. Jenna from PubU remarked how in both softball and in class getting enough practice and feedback was important to become an expert. She stated:

"just practicing and getting help and feedback until you get it right and then you know when you get it right because you're either going to nail the test or you hit the ball"

Chelsea from PriU remarked how practicing art from a much younger age has made her an expert in drawing. She noted that learning in the engineering classroom is different because the concepts are new, and because unlike easier concepts like addition, something as involved as heat transfer engineering is difficult to learn because she has not practiced it for a long time. Similarly, acknowledging the need for repeated practice to gain mastery on concepts, Justin from PriU compared learning tennis to learning in the classroom and reflected:

"I guess just you give yourself the understanding in lecture, or like the basics in lecture and then you go out and you try and do what you learn in lecture. Then if you have a problem with it you come back and say, "Hey I'm not getting this." You work on it again and then until you felt like you master it, and you move on to something else." 
On being asked if he found anything about learning basketball to be drastically different from learning in the classroom, Luis from PriU remarks "Not drastically different, no. I feel like it's just the way that you learn. Practice, practice, practice." Thus, multiple students expressed how practice was a key learning strategy to gain expertise inside or outside the classroom.

Motivation Related Comparisons. Interest in the activity, perceived utility, and relatedness seemed to emerge as basis of comparisons used by students to describe learning in both the settings. In analyzing the statements for both non-academic and academic settings it seemed that students seemed to be motivated by more intrinsic factors such as an interest in the activity or knowing about the usefulness of a concept, or in contrast de-motivated by the lack of immediate usefulness of concepts taught in the class. Out of the seventeen participants, ten used motivation related construct to compare across both the settings. For example, Chris from PriU observes as a major disconnect in academic learning the inability to understand utility of certain concepts, which he is unable to implement or test out. He notes:

That's I suppose one of the big disconnects of a lot of academia stuff. You can't just go out and set things on fire. You can't just start spraying fire hoses everywhere and measuring the fire hoses. That stuff's very expensive. You can't build engines, but people do. Definitely mixing on the hands ... Just having, the "okay, let's go test it out for myself." It's one thing to read something and understand it, but it's one thing to actually say, "Well, okay, I'm going to expose this for a thousandth of a second." Until you can understand what a thousandth of a second is in your own hands, it's just going to be a number to you."

Similarly, Greg from PriU highlights how learning outside the classroom is different because it is self-motivated. He states:

"I think a major difference is that you do it when you want to do it, and nobody is going to measure your progress, it is all self-motivated...I guess as a result of that school becomes your job, and when something is your job it is not fun anymore.

Having a guitar is a hobby. I look forward to learning something new. With school, it grinds you a bit, and maybe it is harder to learn because you are not excited about learning it..."

Conversely, Lisa from PubU explained how her interest in topics taught in the classroom helps her learn in the in-class setting. She compares this to her Zumba dance classes and notes the similarities in her persistence due to interest. She commented:

"The similarities of mechanical engineering, I actually find many class very interesting, because we have learned something about a system and how to approach this and that problem. Those are really fascinating to me sometimes. Zumba, I also have passion for that, because I always think that people can dance is very cool. Even if it's quite difficult at first, but I just feel pretty happy to keep carry on."

Thus, for non-academic settings, students seemed to value learning which suggested intrinsic motivational constructs such as interest and utility whereas for academic settings, a majority of the students primarily seemed to be motivated to learn due to more external factors such as passing the class, good grades, or future jobs. 
Learning from Experts. Four participants compared learning across the settings to have similarities on the basis of expertise. Two of these participants acknowledged the instructor as the expert in the classroom. Jeffrey made a case for similarity for both the settings, and in learning from the experts:

"I don't know. I guess it's similar in the way that you see somebody else do it and it's nice seeing, let's say, a professor works an example. You see how they go through it, you're like, "Okay, that's the method that they used. I like that method, it's organized, I'm going to try and use that too." Then you try and do it"

In comparing the similarities in the outcomes for classroom and outside classroom settings, Robert from PriU noted that just like in football, he found that he was not able to ever be the best, or be as good as the experts. He expressed:

"You can get good at it but you can never be Messi or Christiano Ronaldo at it. That's the people that get 100 on the exams. That how I see it....We only get 15 minutes to shine during that quiz. Sometimes we can't because it's just out of our hands I guess. For me, soccer is the same thing. Sometimes I can't, it's out of my hands. I don't have that much talent and I feel like I do my best. T hat's how I make peace with myself with I get bad grades. If I don't get what I want, if I don't get a ten, I'm like, "Well, I did my best.""'

Conversely, Chelsea from PriU noted that her talent in drawing made her comfortable in learning new techniques and master them for her hobby. However, extending the notion of expertise to her classroom, she reflected that she was not equally talented as a heat transfer engineer, had lower experience or expertise, and hence learning became more involved and difficult.

Use of Technology. Although multiple participants had explained how online forums and blogs helped them learn concepts for hobbies and activities outside the classroom, only two students offered examples of how the use of technology compared across the two settings. Comparing the use of technology for a hobby versus for learning concepts for classrooms, Jerry from PubU, for example, reflected that with assignments it was difficult to figure out which solutions or explanations online are correct since multiple ones are offered. He explained that going to the instructor was the easiest thing to do in order to get the most reliable information. However, on being probed further on whether he used online resources to learn concepts he regularly accesses Khan academy and other reputed tutorials online.

Summarizing Comparisons. We see similarities in the behavioristic approaches to learning through practice and reward in both the settings: inside and outside the classroom. However, important to note are the differences in learning in the two settings. We have described how engineering students often seem to value the coach or expert as a role model in nonacademic settings, however not many students seemed to equate faculty or academic coaches to serve as possible role models in the learning environment inside the classrooms. Additionally, multiple students mentioned technology in helping them understand techniques and principles outside the classroom. However, the role of technology inside the classroom was only highlighted by two participants.

\section{Conclusions and Implications}

The results of our analyses suggest that engineering students view learning inside and outside the classrooms similarly, although some differences exist. The differences in both the 
settings seem to exist primarily due to differences in the causal conditions for learning. While in the classroom, students have no choice or agency on the topics of focus and have to learn all concepts taught, many explain how learning outside the classroom is more interesting because of their own talents and interest in the hobbies they choose.

We believe that this research has implications for engineering students, faculty and engineering education researchers, through helping them understand factors contributing to learning for engineering students in settings both inside and outside the classroom. Faculty and engineering educators can use this understanding of the choices of learning strategies employed by students, and the conditions in which learning happens outside the classroom, and extend it to learning within the classrooms. For instance, the analysis indicates that students were unable to immediately recognize the relevance of topics or concepts taught in the classroom to the real world applications. The converse is true for a hobby, for example while playing tennis, the student is able to appreciate the importance of learning the back-hand stroke since they can immediately put it to use while playing a game. One of the participants expressed how karate was similar to academics in that it taught them the relevance of small, irrelevant parts and how they would eventually contribute to the strength needed for being a black belt. Thus, if instructors are able to indicate the relevance of smaller concepts to the bigger picture for industrial application or day to day experiences, maybe the learner will be more engaged in the classroom. Instructors in the engineering classroom can thus stand to gain immensely from understanding how engineering students approach learning outside their classrooms.

\section{Future Work}

For future work we recommend further interviews through theoretical sampling to gain saturation and for the proposed Grounded Theory model to be truly grounded in all possible perspectives which may be offered through participant views on learning in both settings. Another immediate future step would be to extend this analysis to go beyond the sections of the transcripts offering comparative insight on learning in both settings, to the larger conversation on learning and conceptual understanding. A more involved analysis of how students speak about learning in the classrooms compared to learning outside the classroom may provide insight on specific recommendations for developing learning strategies informed by learning in out of classroom settings which may be of interest to engineering educators. Once a more complete model is created, we could more easily compare and contrast with existing theory.

\section{Acknowledgements}

This paper is based on research supported by the National Science Foundation under Grant Nos. 1150384. Any opinions, findings, and conclusions or recommendations expressed in this material are those of the authors and do not necessarily reflect the views of the National Science Foundation.

\section{References}

Charmaz, K. (2006). Constructing grounded theory. Thousand Oaks, Calif; London: Sage. Creswell, J. W. (2014). Research design: qualitative, quantitative, and mixed method approaches (4th ed.). Thousand Oaks, Calif: Sage Publications.

Creswell, J. W. (2014). A concise introduction to mixed methods research. Sage Publications.

Eccles, J. S., \& Wigfield, A. (2002). Motivational beliefs, values, and goals. Annual review of psychology, 53(1), 109-132. 
Glaser, B. G. (1978). Theoretical sensitivity: advances in the methodology of grounded theory. Mill Valley, Calif U6 Book: Sociology Press.

Glaser, B. G. (1992). Basics of grounded theory analysis: Emergence vs forcing. Sociology Press.

Glaser, B. G., \& Strauss, A. L. (1967). The discovery of grounded theory: strategies for qualitative research. Chicago U6 Aldine Pub. Co.

Heaton, J. (2004). Reworking qualitative data. Thousand Oaks, CA: Sage.

Johri, A., \& Olds, B. M. (2011). Situated engineering learning: Bridging engineering education research and the learning sciences. Journal of Engineering Education, 100(1), 151-185

Jones, B. D. (2009). Motivating students to engage in learning: The MUSIC Model of Academic Motivation. International Journal of Teaching and Learning in Higher Education, 21(2), 272285.

Jones, B., Paretti, M., Hein, S., \& Knott, T. (2010). An analysis of motivation constructs with first-year engineering students: Relationships among expectancies, values, achievement, and career plans. Journal of Engineering Education, 99(4), 319-336. doi:10.1002/j.21689830.2010.tb01066.x

Lempert, L. B. (2007). Asking Questions of the Data. In A. Bryant \& K. Charmaz (Eds.), The SAGE Handbook of Grounded Theory. Thousand Oaks; London: SAGE Publications, Limited.

Leydens, J. A., Moskal, B. M., \& Pavelich, M. (2004). Qualitative methods used in the assessment of engineering education. Journal of Engineering Education, 93(1), 65-72.

Leydens, J. A., Moskal, B. M., \& Pavelich, M. (2004). Qualitative methods used in the assessment of engineering education. Journal of Engineering Education, 93(1), 65-72.

Linnenbrink, E. A., \& Pintrich, P. R. (2002). The role of motivational beliefs in conceptual change. In M. Limon \& L. Mason (Eds.), Reconsidering conceptual change: Issues in theory and practice (pp. 115-135). Dordrecht, The Netherlands: Kluwer Academic.

Matusovich, H. M., Streveler, R. A., \& Miller, R. L. (2010). Why do students choose engineering? A qualitative, longitudinal investigation of students' motivational values. Journal of Engineering Education, 99(4), 289-303.

Matusovich, H., \& McCord, R. (2012). Work in progress: Does motivation matter for conceptual change? exploring the implications of "hot cognition" on conceptual learning. In Frontiers in Education Conference (FIE), 2012 (pp. 1-2). IEEE.

McCord, R., \& Matusovich, H. M. (2013). Developing an Instrument to Measure Motivation, Learning Strategies and Conceptual Change. In 120th ASEE Annual Conference \& Exposition, Atlanta, GA.

Morelock, J., Matusovich, H., Cunningham, P., \& Hermundstad, A. L. (2016). Working the Problems: Creating a Behaviorist Culture. Poster presented at the American Educational Research Association Annual Meeting.

Newstetter, Wendy C., and Marilla D. Svinicki. "Learning theories for engineering education practice." Cambridge handbook of engineering education research (2014): 29-46.

Patton, M. Q., \& Patton, M. Q. (2002). Qualitative research and evaluation methods (3 ed). Thousand Oaks, Calif: Sage Publications.

Phillips, D. C., \& Soltis, J. F. (2004). Perspectives on learning. Teachers College Press.

Pintrich, P. R., Marx, R. W., \& Boyle, R. A. (1993). Beyond cold conceptual change: The role of motivational beliefs and classroom contextual factors in the process of conceptual change. Review of Educational research, 63(2), 167-199. 
Rittle-Johnson, B., Siegler, R. S., \& Alibali, M. W. (2001). Developing conceptual understanding and procedural skill in mathematics: An iterative process. Journal of educational psychology, 93(2), 346.

Robson, C. (2002). Real world research: A resource for social scientists and practitionerresearchers (Vol. 2): Blackwell Oxford.

Steif, P. S., \& Dantzler, J. A. (2005). A statics concept inventory: Development and psychometric analysis. Journal of Engineering Education, 94(4), 363.

Strauss, A. L. (1987). Qualitative analysis for social scientists. Cambridge University Press.

Strauss, A., \& Corbin, J. (1990). Basics of qualitative research (Vol. 15). Newbury Park, CA: Sage.

Strauss, A., \& Corbin, J. (1998). Basics of qualitative research. 1998. Thousand Oaks.

Streveler, R. A., Litzinger, T. A., Miller, R. L., \& Steif, P. S. (2008). Learning conceptual knowledge in the engineering sciences: Overview and future research directions. Journal of Engineering Education, 97(3), 279-294.

Streveler, R. A., Miller, R. L., Santiago-Román, A. I., Nelson, M. A., Geist, M. R., \& Olds, B. M. (2011). Rigorous methodology for concept inventory development: Using the'assessment triangle'to develop and test the thermal and transport science concept inventory (TTCI). International Journal of Engineering Education, 27(5), 968.

Tashakkori, A., \& Teddlie, C. (1998). Mixed methodology: combining qualitative and quantitative approaches (Vol. 46.; 46). Thousand Oaks, Calif: Sage.

Urquhart, C., \& Fernandez, W. (2013). Using grounded theory method in information systems: the researcher as blank slate and other myths. Journal of Information Technology, 28(3), 224236.

Vosniadou, S. (2007). Conceptual change and education. Human Development, 50(1), 47-54. 